\title{
Rifampicin-resistant strains of Mycobacterium leprae may have reduced virulence
}

\author{
R. DENISE MCDERMOTT-LANCASTER and G. R. F. HILSON
}

Department of Medical Microbiology, St. George's Hospital Medical School, Cranmer Terrace, London SW17 ORE

\begin{abstract}
Summary. A strain of Mycobacterium leprae resistant to rifampicin (RMP) failed to infect normal mice when injected into the foot pads (FP) at a dose of 10 or 100 bacilli/ FP, although it could be maintained by serial passage in mice by the use of inocula of $10^{4}$ bacilli/FP; normal mice can be infected by RMP-sensitive $M$. leprae at a dose of 10 bacilli/FP. By contrast, nude (athymic) mice could be infected with an inoculum of 10 bacilli/FP of the RMP-resistant strain. It is suggested that the strain concerned possessed reduced virulence for normal mice, and the implications of this for the probability of occurrence of human disease caused by RMP-resistant strains of $M$. leprae are discussed.
\end{abstract}

\section{Introduction}

Some strains of Staphylococcus aureus and of Mycobacterium tuberculosis that are resistant to rifampicin (RMP) are less virulent for experimental animals than are RMP-sensitive strains of the same species (Daddi et al., 1971 ; Moorman and Mandell, 1981). Since 1976, several strains of $M$. leprae have been found that are RMP-resistant (Jacobson and Hastings, 1976; Guelpa-Lauras et al., 1984), and a chance finding during work with one of these strains suggested that it was less infective for the foot pads of normal mice than are RMP-sensitive strains.

\section{Materials and methods}

These are described briefly. A full description is provided by Lancaster (1985).

\section{Mouse strains}

Normal mice were of the CD1 strain (Charles River Laboratories, Margate, Kent). Nude athymic mice (Harlan Olac Ltd, Bicester, Oxon) were an outbred pathogen-free strain maintained in gnotobiotic isolators. The homozygous $n u / n u$ males used in this work were obtained by breeding from matings of $n u / n u$ males with heterozygous $n u /+$ females.

\section{Strains of M. leprae}

The RMP-resistant strain was described by Jacobson and Hastings (1976) and kindly supplied by Dr R. C. Hastings as a mouse foot-pad (FP) homogenate. It was maintained by serial passage in the FPs of normal mice, with an inoculum of $10^{4}$ acid-fast bacilli (AFB)/FP. This

Received 28 Oct. 1986; accepted 26 Jan. 1987. strain is able to grow in mice given $0.03 \%$ RMP (but not $0.06 \%$ ) in the diet, a resistance 30 times higher than normal. RMP-sensitive strains were obtained from biopsies from patients with leprosy and maintained in the same way. In the experiments reported, the $M$. leprae inocula were in the form of fresh homogenates made from infected FPs.

\section{Inoculation and treatment of mice and harvesting of FPs for AFB counts}

The methods used for inoculation and harvesting of FPs and counting of $M$. leprae in FP homogenates have been described previously (Shepard and McRae, 1968; Lancaster et al., 1983). Reference to "numbers of $M$. leprae" represents total AFB/FP. Administration of RMP was by gastric cannula as a $30 \%(\mathrm{w} / \mathrm{v})$ solution in ethanol, each dose providing $20 \mathrm{mg} / \mathrm{kg}$.

Three groups of five nude mice were inoculated in the right hind FP with $10^{2}, 10^{1}$, and $10^{\circ}$ RMP-resistant $M$. leprae respectively. After an interval of 6 weeks, RMP treatment was started (one dose weekly) and maintained for 41 weeks; three other groups of mice were inoculated similarly but left untreated. Two sets of three groups of normal mice were inoculated similarly, one set being treated as described and the other left untreated. Several mice died at early stages of the experiment; all surviving mice were killed and the FPs removed and homogenised. From the homogenates, microscope slides were prepared for counting AFB. The threshold of detection of $M$. leprae by the method used is about $10^{4} \mathrm{AFB} / \mathrm{FP}$.

\section{Results}

\section{Growth in nude mice}

In the untreated mice, the largest inoculum $\left(10^{2}\right.$ AFB/FP) had reached a mean count of $1.5 \times 10^{8}$ 
AFB/FP at the end of the experiment (table), and growth could have continued further (Colston and Hilson, 1976; Lancaster, 1985). Significant multiplication of the tenfold smaller inoculum $\left(10^{1}\right.$ $\mathrm{AFB})$, to $1.3 \times 10^{6} \mathrm{AFB} / \mathrm{FP}$ in a single surviving mouse, had also occurred. No growth $\left(<10^{4} \mathrm{AFB} /\right.$ FP) was detected in the mice inoculated with the nominal value of $10^{\circ} \mathrm{AFB} / \mathrm{FP}$; furthermore, this was true for all the groups given this inoculum, which presumably did not contain any viable $M$. leprae. In the treated mice given the inoculum of $10^{2} \mathrm{AFB}$, growth had occurred to a mean of $1.8 \times$ $10^{6}$ AFB/FP in three mice. This finding showed that the inoculum still displayed resistance to RMP, but in this group the organisms had grown to a level only $1 \%$ of that in the corresponding group of untreated mice. Growth from the inoculum of $10^{1}$ AFB was apparently also slower; the numbers arising from it only just reached the threshold of detection at the time of harvest.

\section{Growth in normal mice}

No growth was detected from the inocula of $10^{2}$ or $10^{1}$ AFB in 17 mice, whether treated or untreated, whereas prediction from the results in nude mice would have led to an expectation of growth being found in 12 of the 17 .

\section{Discussion}

Growth of the small inocula $\left(10^{2}\right.$ or $10^{1}$ AFB/FP) of RMP-resistant bacilli occurred in the FPs of nude mice, whether treated with RMP or not, as was expected; the apparent retardation of growth

Table. Growth in normal and nude mice of $M$. leprae resistant to rifampicin (RMP).

\begin{tabular}{l|ccc}
\hline & \multicolumn{3}{|c}{ Mean count of acid-fast bacilli/foot pad (AFB/FP) } \\
\cline { 2 - 4 } Mouse & $\begin{array}{c}\text { at start of } \\
\text { experiment } \\
\text { (all mice) }\end{array}$ & RMP-treated & RMP-untreated \\
\cline { 2 - 4 } strain & $10^{2}$ & $1 \cdot 8 \times 10^{6}(3)^{*}$ & $1 \cdot 5 \times 10^{8}(5)$ \\
Nude & $10^{1}$ & $1 \cdot 0 \times 10^{4}(2)$ & $1 \cdot 3 \times 10^{6}(1)$ \\
& $10^{\circ}$ & $<10^{4}(5)$ & $<10^{4}(4)$ \\
Normal & $10^{2}$ & $<10^{4}(4)$ & $<10^{4}(4)$ \\
& $10^{1}$ & $<10^{4}(5)$ & $<10^{4}(4)$ \\
& $10^{\circ}$ & $<10^{4}(4)$ & $<10^{4}(4)$ \\
\hline
\end{tabular}

* The number in parenthesis is the number of foot pads harvested on which the number of AFB/FP was based. of the resistant strain in RMP-treated mice could have reflected actual slowing of growth of the RMP-resistant bacilli, or could have been due to the unsuspected presence in the inoculum of a proportion of RMP-sensitive bacilli which contributed to the growth in the untreated mice. The finding of interest was that there was no evidence of growth of the small inocula in any of the 17 surviving normal mice. It is well known that $10^{2}$ AFB of RMP-sensitive $M$. leprae will regularly initiate growth in FPs of normal mice (Shepard, 1960; Shepard and McRae, 1965), and we have found that such small inocula will also show a normal growth rate in nude mice (Lancaster et al., 1983; Lancaster, 1985). It appeared, therefore, that the RMP-resistant strain resembled sensitive strains in growing in the FPs of normal mice from inocula of $5 \times 10^{3}$ AFB (Jacobson and Hastings, 1976) or $10^{4} \mathrm{AFB}$ and in growing from an inoculum of $10^{2}$ AFB in nude mice, but differed in that it did not yield detectable growth from the latter inoculum after 41 weeks in the FPs of normal mice. We cannot say whether there was no multiplication at all of the inoculum, or whether growth was occurring, but too slowly for the threshold of detection to be reached by the time the experiment was concluded. In either case, the results suggest that the RMP-resistant strain was at a disadvantage by comparison with sensitive strains in the face of the relatively high cellular immune response of normal mice, which is lacking in nude mice. Further work will be needed to confirm this suggestion; in this, the minimum infective dose and growth curve of this and other RMP-resistant strains of $M$. leprae would be compared with those of sensitive strains.

In pathogenic mycobacteria, drug resistance is mediated by chromosomal mutation, and strains derived in this way may show reduced virulence in experimental animals (e.g., isoniazid resistance of $M$. tuberculosis; Meissner, 1964). However, it is well known that clinical disease may be initiated by strains of tubercle bacilli resistant to streptomycin, isoniazid or $p$-aminosalicylic acid (primary drug-resistant tuberculosis), which demonstrates that significant virulence for man is retained by them. Similarly, dapsone-resistant strains of $M$. leprae can cause leprosy, including the tuberculoid variety characterised by relatively high cellular immunity (Waters and Laing, 1978). Reduced virulence of RMP-resistant strains of $M$. tuberculosis for mice or guinea-pigs has been reported by several authors, e.g., an inoculum of $0.5 \mathrm{mg}$ in mice is not lethal, unlike a similar inoculum of a sensitive wild strain (Daddi et al., 1971). However, some 
RMP-resistant strains may retain virulence for man because at least 12 cases of primary RMP-resistant tuberculosis have been reported (Kopanoff et al., 1978; Steiner et al., 1983).

The RMP-resistant strain of $M$. leprae described here was isolated from a nodule which developed on the skin of a lepromatous patient who was responding well to RMP treatment (Jacobson and Hastings, 1976), showing that the mutant had the capacity to grow in the presence of the low level of cellular immunity to $M$. leprae found in such patients. If reduced mouse-virulence of RMP-

\section{REFERENCES}

Colston M J, Hilson G R F 1976 Growth of Mycobacterium marinum and Mycobacterium leprae in congenitally athymic (nude) mice. IRCS Medical Science 4: 186.

Daddi G, Lucchesi M, Mancini P, Termine A 1971 Virulence attenuation and other biological modifications in multiple drug-resistant tubercle mycobacteria. Bolletino Istituto Sieroterapico Milanese 50: 353-361.

Guelpa-Lauras C, Grosset J H, Constant-Desportes M, Brucker G 1984 Nine cases of rifampin-resistant leprosy. International Journal of Leprosy 52: 101-102.

Jacobson R R, Hastings R C 1976 Rifampin-resistant leprosy. Lancet 2: 1304-1305.

Kopanoff D E, Kilburn J O, Glassroth J L, Snider D E, Farer L S, Good R C 1978 A continuing survey of tuberculosis primary drug resistance in the United States: March 1975 to November 1977. A United States Public Health Service Cooperative Study. American Review of Respiratory Disease 118: $835-842$.

Lancaster R D 1985 The development and use of the nude mouse as a model of lepromatous leprosy. PhD thesis, University of London.

Lancaster R D, Hilson G R F, McDougall A C, Colston M J 1983 Mycobacterium leprae infection in nude mice: bacteri- resistant strains of $M$. leprae is confirmed, there would be an implication that the risk of occurrence of primary RMP-resistant leprosy would also be relatively reduced, and the disease would present at the lepromatous end of the spectrum. No case of primary RMP-resistant leprosy has yet been reported, but it is probably too soon to expect one.

This investigation was supported by the Chemotherapy of Leprosy component of the United Nations Development Programme/World Bank/World Health Organization Special Programme for Research and Training in Tropical Diseases TRR/AR(1)/77.16b.

ological and histological responses to primary infection and large inocula. Infection and Immunity 39: 865-872.

Meissner G 1964 The bacteriology of the tubercle bacillus. In: Barry V C (ed) Chemotherapy of tuberculosis. Butterworths, London, pp 80-89.

Moorman D R, Mandell G L 1981 Characteristics of rifampinresistant variants obtained from clinical isolates of Staphylococcus aureus. Antimicrobial Agents and Chemotherapy 20 : 709-713.

Shepard C C 1960 The experimental disease that follows the injection of human leprosy bacilli into foot-pads of mice. Journal of Experimental Medicine 112: 445-454.

Shepard C C, McRae D H 1965 Mycobacterium leprae in mice: minimal infectious dose, relationship between staining quality and infectivity, and effect of cortisone. Journal of Bacteriology 89 : 365-372.

Shepard C C, McRae D H 1968 A method for counting acidfast bacteria. International Journal of Leprosy 36: 78-82.

Steiner P, Rao M, Victoria M S, Hunt J, Steiner M 1983 A continuing study of primary drug-resistant tuberculosis among children observed at the Kings County Hospital Medical Center between the years 1961 and 1980. American Review of Respiratory Disease 128: 425-428.

Waters M F R, Laing A B G, Rees R J W 1978 Proven primary dapsone resistance in leprosy - a case report. Leprosy Review 49: $127-130$. 